\title{
The Change in Regional Cerebral Oxygen Saturation after Stellate Ganglion Block
}

\author{
${ }^{1}$ Department of Anesthesiology and Pain Medicine, \\ ${ }^{2}$ Anesthesia and Pain Research Institute, Yonsei University College of Medicine, Seoul, \\ ${ }^{3}$ Department of Anesthesiology and Pain Medicine, CHA Bundang Medical Center, \\ CHA University, Seongnam, Korea \\ Hyeon Min Park, MD ${ }^{1,2}$, Tae Wan Kim, MD ${ }^{3}$, Hong Gyu Choi, MD', \\ Kyung Bong Yoon, $\mathrm{MD}^{1,2}$, and Duck Mi Yoon, $\mathrm{MD}^{1,2}$
}

\section{Background:}

Stellate ganglion block (SGB) is known to increase blood flow to the innervations area of the stellate ganglion. Near infrared spectroscopy reflects an increased blood volume and allows continuous, non-invasive, and bedside monitoring of regional cerebral oxygen saturation $\left(\mathrm{rSO}_{2}\right)$. We investigated the influence of SGB on bilateral cerebral oxygenation using a near infrared spectroscopy.

\section{Methods:}

SGB was performed on 30 patients with $1 \%$ lidocaine $10 \mathrm{ml}$ using a paratracheal technique at the C6 level and confirmed by the presence of Horner's syndrome. The blood pressure (BP), heart rate (HR) and $\mathrm{rSO}_{2}$ were measured before SGB and 5, 10, 15 and 20 minutes after SGB. Tympanic temperature of each ear was measured prior to SGB and 20 minutes after SGB.

\section{Results:}

The increments of the $\mathrm{rSO}_{2}$ on the block side from the baseline were statistically significant at $5,10,15$ and 20 minutes. The $\mathrm{rSO}_{2}$ on the non-block side compared with the baseline, however, decreased at 15 and 20 minutes. The difference between the block and the non-block sides was significant at 15 and 20 minutes. The $\mathrm{BP}$ at 10, 15 and 20 minutes was increased and the $\mathrm{HR}$ was increased at 10 and 15 minutes.

\section{Conclusions:}

We observed an increment of the $\mathrm{rSO}_{2}$ on the block side from the baseline; however, the $\mathrm{rSO}_{2}$ on the non-block side decreased. (Korean J Pain 2010; 23: 142-146)

\section{Key Words:}

near infrared spectroscopy, regional cerebral oxygen saturation, stellate ganglion block.

Received December 17, 2009. Revised January 7, 2010. Accepted February 26, 2010.

Correspondence to: Duck Mi Yoon, MD

Department of Anesthesiology and Pain Medicine, Anesthesia and Pain Research Institute, Yonsei University College of Medicine, 134 Shinchon-dong, Seodaemun-gu, Seoul 120-752, Korea

Tel: +82-2-2228-2420, Fax: +82-2-312-7185, E-mail: dmyoon@yuhs.ac

() This is an open-access article distributed under the terms of the Creative Commons Attribution Non-Commercial License (http:// creativecommons.org/licenses/by-nc/3.0/), which permits unrestricted non-commercial use, distribution, and reproduction in any medium, provided the original work is properly cited.

Copyright (C) The Korean Pain Society, 2010 


\section{INTRODUCTION}

Stellate ganglion block (SGB) is often facilitated to improve blood flow and reduce pain in the head, neck, and upper extremity because it entails blocking the cervicothoracic sympathetic ganglion, which is the inferior cervical sympathetic ganglion and the first thoracic sympathetic ganglion in fused form $[1,2]$.

Autoregulation of the cerebral blood flow is mediated by metabolic and chemical factors [3]. Although the effect of sympathetic nerve stimulation or block on the cerebral blood flow remains a subject of debate, literature on human studies reports an increased cerebral blood flow after SGB $[4,5]$. One method of evaluating the adequacy of cerebral blood flow may be executing a neurologic examination on conscious patients. Other means of examination include monitoring of the somatosensory evoked potential, jugular venous oxygen saturation, stump pressure, mesencephalic artery blood flow via transcranial doppler, and cerebral oxygen saturation [6]. The four methods mentioned above require expertise to perform, as well as additional devices, are less feasible to use in routine clinical practice and are costly. On the other hand, measuring the regional cerebral oxygen saturation $\left(\mathrm{rSO}_{2}\right)$ at the frontal lobe of the brain cortex by means of near infrared spectroscopy is a simple, non-invasive method which may reflect the cerebral blood flow $[7,8]$.

In this present study, we compared the regional cerebral oxygen saturations at the stellate ganglion block side (block side) to those of the contralateral side where SGB was not performed (non-block side) to investigate the influence SGB may have on the regional cerebral oxygen saturation.

\section{MATERIALS AND METHODS}

After obtaining an approval from the institutional review board, 30 patients who were scheduled to receive stellate ganglion block at our pain management clinic were recruited between July and September 2009. Written informed consent was obtained from all patients. Inclusion criteria were ages between 20 and 65 years, disease entity in the head, neck, and upper extremity, and ASA PS 1, 2. Patients with the tendency to bleed and who had cerebrovascular disease were excluded from this study.

Patients were positioned supine with a shallow pillow under the shoulder. Regional cerebral oxygen saturation, blood pressure (BP), heart rate (HR) and the tympanic temperature of each ear were obtained 5 minutes after monitoring with a near infrared spectroscopy (INVOS 5100, Somanetics, Troy, USA), a non-invasive blood pressure manometer, an electrocardiogram and a pulse oximeter. SBG was performed with an anterior paratracheal approach at the $\mathrm{C} 6$ transverse process level and $1 \%$ lidocaine $10 \mathrm{ml}$ was injected.

Regional cerebral oxygen saturation, BP and HR were measured at the baseline and at 5 minute intervals for 20 minutes (5, 10, 15, 20 minutes), and the tympanic temperature was measured twice-before and 20 minutes after the block at both ears. A successful SGB was determined based on the block symptoms and sign such as ptosis, miosis, injection of the sclera and conjunctiva, nasal stiffness, and facial hot flush 15 minutes after SGB.

Statistic analysis was performed using SPSS 15.0 (SPSS Inc., Chicago, IL, USA). Data were shown as mean \pm standard deviation. Comparisons of the block side and non-block side at each time point were performed using a paired t-test with Bonferroni post hoc comparisons. Comparisons of the parameters at each time point with the baseline values were analyzed using one way repeated-measures ANOVA and Dunnet's test. $P<0.05$ was considered statistically significant.

\section{RESULTS}

Thirty patients, 7 male and 23 female, with the mean age of $48.3 \pm 14.7$ years, were enrolled in this study. The block side of SGB was on the right in 10 patients and on the left in 20 patients. Presentation of the patients' symptoms were facial pain in 9 patients, neck pain in 6 , shoulder pain in 4, upper extremity pain in 4, sudden onset sensory neural hearing loss in 3 , headache in 3 and toothache in 1 patient. All patients showed symptoms and signs of successful SGB.

Blood pressure before and after SGB was significantly increased at 10, 15 and 20 minutes, and heart rate was significantly increased at 10 and 15 minutes (Fig. 1). The tympanic temperature changes were not significant before and after the block.

Regional cerebral oxygen saturations on the block side and the non-block side were significantly different at 15 and 20 minutes. In comparison to the baseline values, 
A

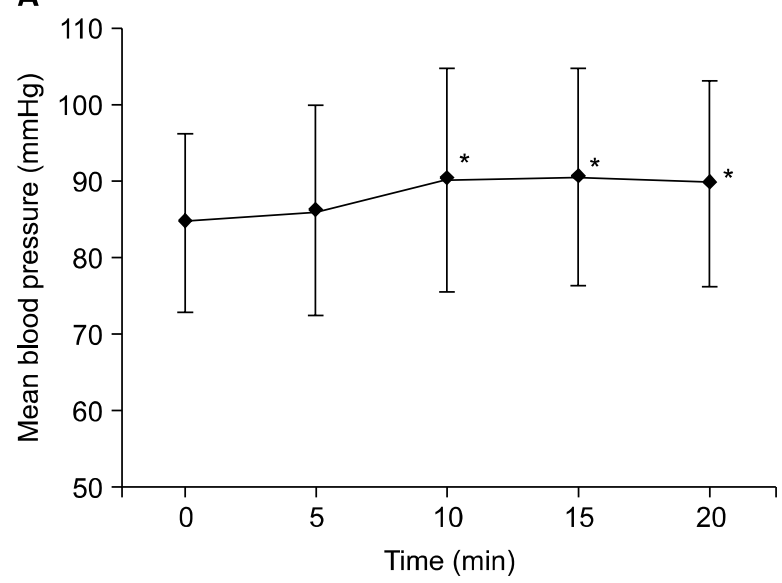

B

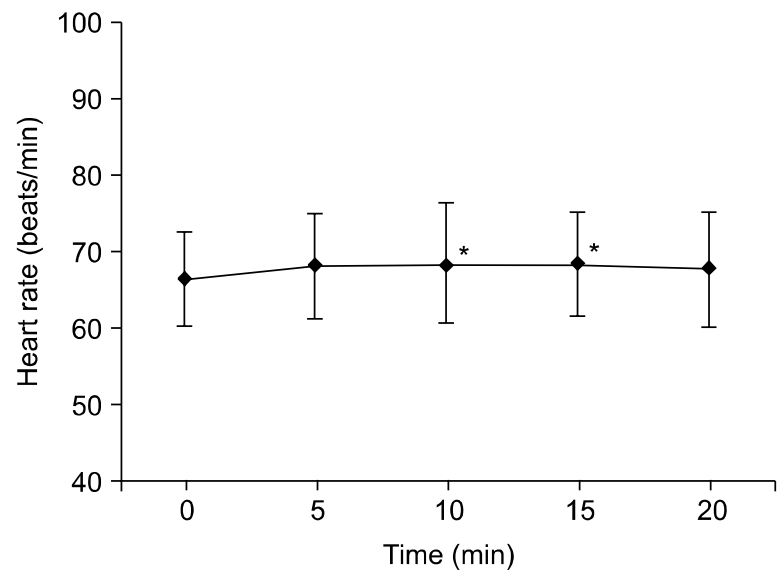

Fig. 1. Changes in mean blood pressure (mBP; $A$ ) and heart rate (HR; B) were assessed for 20 minutes, at 5 minute intervals after completion of stellate ganglion block. The $\mathrm{mBP}$ and HR were increased as compared with the baseline values. Data are expressed as mean $\pm \mathrm{SD}$. ${ }^{\star} P<0.05$ as compared with the baseline values in $(\mathrm{A}, \mathrm{B})$.

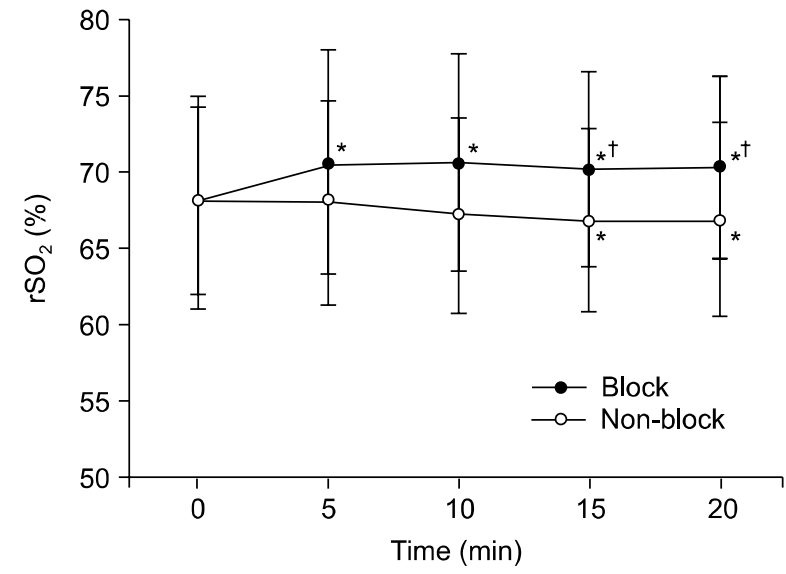

Fig. 2. Changes in regional cerebral oxygen saturations $\left(\mathrm{rSO}_{2}\right)$ were assessed for 20 minutes, at 5 minute intervals after completion of stellate ganglion block. The $\mathrm{rSO}_{2}$ from the baseline on the block side increases significantly, but on the non-block side decreases slightly. Data are expressed as mean $\pm \mathrm{SD}$. ${ }^{\star} P<0.05$ as compared with the baseline values and ${ }^{\dagger} P<0.01$ as compared between the block side and non-block side.

there were significant increases on the block side at 5, 10, 15 and 20 minutes and significant decreases on the non-block side at 15 and 20 minutes (Fig. 2).

\section{DISCUSSION}

Stellate ganglion block is considered successful if resultant symptoms and signs, such as Horner's syndrome (ptosis, miosis, sclera and conjunctival injection) and increase of the skin temperature at the ipsilateral side, develop $[9,10]$. These clinical evaluations are commonly used in clinical practice to distinguish a successful SGB. In recent years, however, more direct and objective means of its evaluation have been developed, which include measuring the changes in the cerebral blood flow using MRI, CT and transcranial Doppler ultrasonography [11,12]. On the other hand, cerebral oxygen saturation monitoring using near infrared spectroscopy has also been introduced as having advantages over the cerebral blood flow monitorings regarding its non-invasiveness and easy feasibility.

Cerebral oxygen saturation monitoring, first introduced by Jobsis, is a new and non-invasive method to continuously monitor and detect regional cerebral oxygen saturation of the frontal lobe of the brain cortex by attaching a sensor on the forehead [13].

The sensor detects and calculates the ratio of oxyhemoglobin to total hemoglobin by using near infrared spectropscopy [14,15]. The cerebral oximeter measures hemoglobin oxygen saturation in the tissues up to the capillary, arteriole and venular level by using optical density, which differentiates it from the pulse oximeter that detects arterial oxygen saturation by obtaining a pulsatile signal of the arterial blood flow.

Cerebral blood from the brain tissue is $75 \%$ venous and $25 \%$ either arterial or capillary. Near infrared spectroscopy used in this study has 2 sensors that subtract the optical absorption of the superficial areas from the deep 
areas [14-16] and are known to show a mean of $\mathrm{rSO}_{2}$ of $68 \pm 5.6 \%$ in normal adults and no difference regarding age and sex [17].

Central venous oxygen saturation $\left(\mathrm{ScvO}_{2}\right)$ linearly reflects cerebral blood flow if the cerebral metabolic rate is constant, and $\mathrm{ScvO}_{2}$ can replace jugular bulb venous oxygen saturation $\left(\mathrm{SjO}_{2}\right)$. In previous studies, $\mathrm{rSO}_{2}$ is reported to have a good correlation with $\mathrm{SjO}_{2}[7,8]$. One study relates a good correlation between $\mathrm{rSO}_{2}$ and $\mathrm{SjO}_{2}$ when normal ventilation and hyperventilation were strictly controlled based on the arterial $\mathrm{CO}_{2}$ tension in normal adults [18]. Some studies postulated that changes in the cerebral blood flow may have a great influence on $\mathrm{rSO}_{2}[19,20]$ because regional cerebral oxygen saturation reflects regional oxygen saturation.

In the present study, the $\mathrm{rSO}_{2}$ on the block side increased, which may be considered as reflecting the increased cerebral blood flow after SGB. The decreased $\mathrm{rSO}_{2}$ on the non-block side may be explained as a phenomenon of blood shifting from the non-block side to the block side. This phenomenon may be similar to the steal phenomenon, which is the cerebral blood flow moving from the focal stenosed cerebral vessels to the normal cerebral vessels in vasodilatory status [21].

Using local anesthetics to perform SGB may affect the cardiovascular system, causing changes in the BP and HR. There is a case report of severe hypertension after SGB [22]. However, contrasting results also exist, indicating that SGB does not affect the BP or HR [23]. The present study showed that the BP and HR increased without clinical significance. Increased cardiac output due to increased BP and HR affect both the block side and non-block side; therefore, interpretation of the study's result, which shows an increased difference in $\mathrm{rSO}_{2}$ between the block side and the non-block side, is not confounded by the changes in hemodynamic profile.

Vasodilation after SGB and increased cerebral blood flow may cause a reflux heat exchange between the carotid artery and the jugular vein, potentially leading to brain cooling and decreased tympanic temperature on the block side [24], but this study showed no significant changes to the tympanic temperature of both ears before and after the block.

In conclusion, after $\mathrm{SGB}$, regional cerebral oxygen saturation increased on the ipsilateral block side and decreased on the contralateral non-block side. Decreased
$\mathrm{rSO}_{2}$ on the non-block side may warrant further investigation.

\section{REFERENCES}

1. Song SO, Jo YW. Effects of the volume of local anesthetic used in stellate ganglion block on the elevation of skin temperature of ipsilateral upper extremity. Korean $J$ Anesthesiol 1999; 37: 233-9.

2. Seo YS, Kim SH, Hur CR, Lee KJ, Lee SY, Kim CH, et al. Changes in blood flow velocity of middle cerebral artery after stellate ganglion block. Korean J Pain 1996; 9: 57-62.

3. Patel PM, Drummond JC. Cerebral physiology and the effects of anesthetics and techniques. In: Miller's anesthesia. 6th ed. Edited by Miller RD. Philadelphia, Churchill Livingstone Publishers. 2005, pp 813-20.

4. Umeyama T, Kugimiya T, Ogawa T, Kandori Y, Ishizuka A, Hanaoka K. Changes in cerebral blood flow estimated after stellate ganglion block by single photon emission computed tomography. J Auton Nerv Syst 1995; 50: 339-46.

5. Okubo Y, Ogata H. Brain blood volume measured with near infrared spectroscopy increased after stellate ganglion block. Masui 1995; 44: 423-7.

6. Mahla ME, Susan B, Cucchiara RF. Neurologic monitoring. In: Miller's anesthesia. 6th ed. Edited by Miller RD. Philadelphia, Churchill Livingstone Publishers. 2005, pp 1540-2.

7. Olsen KS, Svendsen LB, Larsen FS. Validation of transcranial near-infrared spectroscopy for evaluation of cerebral blood flow autoregulation. J Neurosurg Anesthesiol 1996; 8: 280-5.

8. Henson LC, Calalang C, Temp JA, Ward DS. Accuracy of a cerebral oximeter in healthy volunteers under conditions of isocapnic hypoxia. Anesthesiology 1998; 88: 58-65.

9. Stevens RA, Stotz A, Kao TC, Powar M, Burgess S, Kleinman $B$. The relative increase in skin temperature after stellate ganglion block is predictive of a complete sympathectomy of the hand. Reg Anesth Pain Med 1998; 23: 266-70.

10. Lee HK, Chung SY, Yang SK, Lee HJ, Suh YS, Kim C. Minimal volume of local anesthetic for successful stellate ganglion block. Korean J Pain 1995; 8: 60-4.

11. Gupta MM, Bithal PK, Dash HH, Chaturvedi A, Mahajan RP. Effects of stellate ganglion block on cerebral haemodynamics as assessed by transcranial Doppler ultrasonography. $\mathrm{Br} J$ Anaesth 2005; 95: 669-73.

12. Nitahara K, Dan K. Blood flow velocity changes in carotid and vertebral arteries with stellate ganglion block: measurement by magnetic resonance imaging using a direct bolus tracking method. Reg Anesth Pain Med 1998; 23: 600-4.

13. Jöbsis FF. Noninvasive, infrared monitoring of cerebral and myocardial oxygen sufficiency and circulatory parameters. Science 1977; 198: 1264-7.

14. Dujovny M, Ausman Jl, Stoddart H, Slavin KV, Lewis GD, 
Widman R. Somanetics INVOS 3100 cerebral oximeter. Neurosurgery 1995; 37: 160.

15. McCormick PW, Stewart M, Goetting MG, Dujovny M, Lewis G. Ausman Jl. Noninvasive cerebral optical spectroscopy for monitoring cerebral oxygen delivery and hemodynamics. Crit Care Med 1991; 19: 89-97.

16. Williams $\mathbb{M}$, Picton A, Farrell A, Mead GE, Mortimer AJ, McCollum CN. Light-reflective cerebral oximetry and jugular bulb venous oxygen saturation during carotid endarterectomy. Br J Surg 1994; 81: 1291-5.

17. Slavin KV, Dujovny M, Ausman Jl, Hernandez G, Luer M, Stoddart $H$. Clinical experience with transcranial cerebral oximetry. Surg Neurol 1994; 42: 531-9.

18. Lee YS, Kwon TM, In JY, Woo SH, Yon JH, Kim JW, et al. Reliability of $\mathrm{rSO}_{2}$ to measure $\mathrm{CO}_{2}$ reactivity of cerebral vasculatures during desflurane and $\mathrm{N}_{2} \mathrm{O}$ anesthesia. Korean $\mathrm{J}$ Anesthesiol 2002; 43: 288-93.

19. Duncan LA, Ruckley CV, Wildsmith JA. Cerebral oximetry: a useful monitor during carotid artery surgery. Anaesthesia
1995; 50: 1041-5

20. Daubeney PE, Pilkington SN, Janke E, Charlton GA, Smith DC, Webber SA. Cerebral oxygenation measured by near Infrared spectroscopy: comparison with jugular bulb oximetry. Ann Thorac Surg 1996; 61: 930-4.

21. Norris EJ. Anesthesia for vascular surgery. In: Miller's anesthesia. 7th ed. Edited by Miller RD. Philadelphia, Churchill Livingstone Publishers. 2009, pp 2030-1.

22. Kimura T, Nishiwaki K, Yokota S, Komatsu T, Shimada Y. Severe hypertension after stellate ganglion block. $\mathrm{Br} J$ Anaesth 2005; 94: 840-2.

23. Goh JS, Min BW, Kim HD. Blood pressure, pulse rate and temperature changes of the ipsilateral upper extremity after unilateral stellate ganglion block. Korean J Pain 1990; 3: 27-33.

24. Murakawa K, Noma K, Ishida K, Matsuda M, Maeda S, Nishimura $\mathrm{M}$, et al. Changes of tympanic temperature by stellate ganglion block. Masui 1995; 44: 824-7. 